\title{
PAPR Reduction of OFDM Signal Using Sequential Phase Sequence SLM Based Transceiver without Side Information
}

\author{
Md Salim Raja ${ }^{a}$, Md. Imran Hossain ${ }^{b}$, Md. Khalid Hossain Jewel ${ }^{c}$, Md Shahjahan Ali $^{\mathrm{c}^{*}}$ \\ ${ }^{a}$ Electronic and Telecommunication Engineering, Hamdard University, Dhaka, Bangladesh \\ ${ }^{b}$ Information and Communication Engineering, Pabna Science and Technology University, Pabna, Bangladesh \\ ${ }^{c}$ Applied Physics, Electronics and Communication Engineering, Islamic University, Kushtia, Bangladesh
}

\begin{abstract}
Researches in the reduction of peak-to-average power ratio (PAPR) from orthogonal frequency division multiplexing (OFDM) output signals are being done using many techniques. Selected mapping (SLM) technique is a distortion-less technique and is a well known method to reduce high PAPR of OFDM signal. One drawback of SLM is its requirement of side information bits to recover original data block at the receiver side which lead rate of data loss. In this paper, we have proposed a sequential phase sequence SLM (SPS-SLM) based OFDM transceiver without side information and studied its performance. For the proposed method, a specific set of phase sequences is designed. MATLAB simulation shows that the proposed scheme performs well in terms of PAPR. Proposed method provides almost the same PAPR reduction performance compared to the conventional SLM method requiring side information.
\end{abstract}

Index Terms: OFDM, Selected mapping technique, Partial transmit sequence, Peak to average power ratio, Additive white Gaussian noise channel, Complimentary cumulative distribution function.

(C) 2015 Published by MECS Publisher. Selection and/or peer review under responsibility of the Research Association of Modern Education and Computer Science

\section{Introduction}

OFDM system is an attractive transmission technique for high speed data communication that offers a considerable high spectral efficiency, multipath delay spread tolerance, immunity to the frequency selective fading channels and power efficiency [1-2].

One of the major problems of OFDM signal is the high PAPR which leads to power inefficiency in RF section of the transmitter and increased complexity in the analog to digital and digital to analog conversion.

\footnotetext{
* Corresponding author. Tel.: +880 71 (PABX) 74910-21 Ext 2270; fax: +880 7174905

E-mail address: jahanshah.iu@gmail.com, msa@aece.iu.ac.bd
} 


\begin{tabular}{|ll|}
\hline Nomenclature & \\
AWGN & additive white Gaussian noise \\
CCDF & complementary cumulative distribution function \\
FFT & fast Fourier transform \\
IFFT & inverse fast Fourier transform \\
ML & maximum likelihood \\
OFDM & orthogonal frequency division multiplexing \\
P/S & parallel to serial \\
PSK & phase shift keying \\
PAPR & peak-to-average power ratio \\
QAM & quadrature amplitude modulation \\
QPSK & quadrature phase shift keying \\
SLM & sequential phase sequence \\
S/P & serial to parallel \\
TI & tone injection \\
TR & tone reservation \\
\hline
\end{tabular}

The distortion and distortion-less (scrambling) technique has been developed to overcome high PAPR problem of OFDM signals [3], [4], [5], [6-7], [8-9]. Selected mapping (SLM) is one of the most promising among all these techniques because it is simple to implement, introduces no distortion in the transmitted signal, and can achieve significant PAPR reduction [2]. With this technique, the price to pay is a loss in data rate due to the transmission of several side information bits. To avoid the need for explicit side information transmission in SLM, a few techniques have been proposed such as the scrambling method described in [10], partial transmission sequence (PTS) [11] and the maximum likelihood (ML) decoding scheme introduced in [12]. In this paper, we have proposed a sequential phase sequence SLM (SPS-SLM) based OFDM transceiver which recover original transmitted data block requiring no side information.

The remainder of this paper is organized as follows. In section II OFDM systems, its problems and solution techniques are given. Section III describes the proposed sequential phase sequence (SPS-SLM) based OFDM transceiver. In Section IV results of the simulation are provided. Section V gives the conclusion of the study.

\section{OFDM Systems, its Problems and Solution Techniques}

In OFDM system frequency bandwidth, $B$, is divided into $N$ orthogonal subcarriers of bandwidth $\Delta f$; where $B$ $=N \Delta f$. In OFDM transmitter, the serial input data stream is divided into frames of $\mathrm{N}_{\mathrm{f}}$ bits. These $\mathrm{N}_{\mathrm{f}}$ bits are arranged into $\mathrm{N}$ groups, where $\mathrm{N}$ is the number of subcarriers. The number of bits in each of the $\mathrm{N}$ groups determines the constellation size for that particular subcarrier. For example, if all the subcarriers are modulated by quadrature phase shift keying (QPSK) then each of the groups consists of 2 bits, whereas in 16-QAM modulation each group contains 4 bits. A block of $N$ frequency domain subcarriers can be denoted as a vector $\mathrm{X}=\left[X_{0}, X_{1} \ldots \ldots . \ldots, X_{N-1}\right]$. In the time domain, via an inverse fast Fourier transform (IFFT) operation one can obtain $x=\left[x_{0}, x_{1} \ldots \ldots, x_{N-1}\right]$. Thus, the sampled sequence can be expressed as the following equation [13].

$$
x(n)=\frac{1}{N} \sum_{k=0}^{N-1} X[k] e^{j 2 \pi K \frac{1}{N} n}
$$

The OFDM receiver executes the reverse operation of OFDM transmitter to recover every original transmitted data blocks. PAPR for the OFDM signal in the time domain $\mathrm{x}$ may be expressed as: 


$$
\mathrm{PAPR}=\frac{P_{\text {peak }}}{P_{\text {average }}}=\frac{\max \left[\left|x_{n}\right|^{2}\right]}{E\left[\left|x_{n}\right|^{2}\right]}=\frac{\max \left[|x(n)|^{2}\right]}{E\left[|x(n)|^{2}\right]}
$$

PAPR is measured in decibel $(\mathrm{dB})$ and can be defined as

$$
\operatorname{PAPR}(\mathrm{db})=10 \log _{10} \frac{\left.\max [\mid x(n)]^{2}\right]}{\left.E[\mid x(n)]^{2}\right]}
$$

PAPR reduction methods can be classified into distortion-less (redundancy or scrambling) and distortion techniques. Distortion techniques are considered to introduce spectral re-growth. They do not require any side information to be sent and they have low complexity compared to the distortionless techniques. On the other hand, distortionless techniques do not introduce spectral regrowth but they require sending side information. The distortion-based techniques reduce the PAPR of the OFDM symbol with the price of adding distortion to the signal points in the subcarriers. Direct clipping [14] simply suppresses the time-domain OFDM signals of which the signal powers exceed a certain threshold. The penalty is the significant increase of out-of-band energy. Peak windowing [15] or filtering after direct clipping [7] can be used to reduce the out of-band energy. After the filtering operation, the peak of the time-domain signal may regrow. Hence, recursive clipping and filtering (RCF) [16] can be used to suppress both the out-of-band energy and the PAPR. RCF can be modified by restricting the region of distortion [17] to obtain improved error performance. On the other hand, estimation of the clipping noise at the receiver can be used to improve the error performance of direct clipping or RCF [9]. The redundancy-based technique includes coding, selective mapping (SLM), partial transmit sequences (PTS), tone reservation (TR) and tone injection (TI), [10], [12], [18], [19], [20-21], [22] etc. A block coding technique [23] is to transmit only the code words with low PAPR. Such coding techniques offer good PAPR reduction performance and coding gain. Significant advances of the coding approach for PAPR control using generalized Reed-Muller codes is summarized in [24]. The critical problem for the coding approach is that for the OFDM system with large number of subcarriers, either it encounters design difficulties or the consequent coding rate becomes prohibitively low. The basic idea of SLM technique is to generate several OFDM symbols as candidates and then select one with the lowest PAPR for actual transmission. Conventionally, the transmission of side information is needed so that the receiver can use the side information to tell which candidate is selected in the transmission.

\section{Proposed Sequential Phase Sequence (SPS-SLM) based OFDM Transceiver}

In SPS-SLM based OFDM Transmitter, the random binary data is considered as data source. The random binary data is converted into symbolic form. The number of binary data per symbol depends on modulation technique applied. The serial symbolic data are divided into a number of blocks. Every block contain $\mathrm{N}$ data symbols which is equal to the number of subcarriers that was taken for OFDM transmitter. The $\mathrm{N}$ serial symbols in every data block converted into $\mathrm{N}$ parallel symbols in each data block by serial to parallel (S/P) converter. $\mathrm{N}$ parallel symbols in every data block again converted into $\mathrm{N}$ complex form symbol using mapping (such as M-array QAM or PSK, etc). The model of SPS-SLM based OFDM transmitter is shown in Fig.(1).

A block of $N$ frequency domain symbol can be denoted as a vector $\mathrm{X}_{\mathrm{n}}=\left[X_{0}, X_{1} \ldots \ldots \ldots, X_{N-1}\right]^{\mathrm{T}}$. Each block is multiplied (element by element multiplication) by $U$ different phase sequences vectors $\mathrm{P}^{(\mathrm{u})}=\left[\mathrm{P}_{0}^{(\mathrm{u})}, \mathrm{P}^{(\mathrm{u})}{ }_{1}\right.$, $\left.\mathrm{P}^{(\mathrm{u})}{ }_{2} \ldots \ldots \ldots \ldots . . . \mathrm{P}^{(\mathrm{u})}{ }_{\mathrm{N}-1}\right]^{\mathrm{T}}$, where $\mathrm{u}=1,2,3 \ldots \mathrm{U}$. In this study $\mathrm{P}^{(\mathrm{u})}$ has been denoted as sequential phase sequence (SPS) whose characteristics can be described by 


$$
\begin{aligned}
& \mathrm{P}^{(\mathrm{u})}=\mathrm{p}_{\mathrm{k}}{ }^{(\mathrm{u})}=\exp \left(\mathrm{j} \phi_{\mathrm{k}}{ }^{(\mathrm{u})}\right) \\
& \mathrm{P}_{\mathrm{k}}{ }^{(\mathrm{u})}=\exp [\mathrm{j}\{2 \pi \mathrm{k}(\mathrm{u}-1) / \mathrm{D}+2 \pi(\mathrm{u}-1) / \mathrm{D}\}]
\end{aligned}
$$

where, $\mathrm{k}$ represents the IFFT point and $\mathrm{D}$ is called phase divider whose value is equal to the integer multiple of the number of sub carriers. In this research work, we have considered the value of $\mathrm{D}$ equal to two times the number of subcarriers.

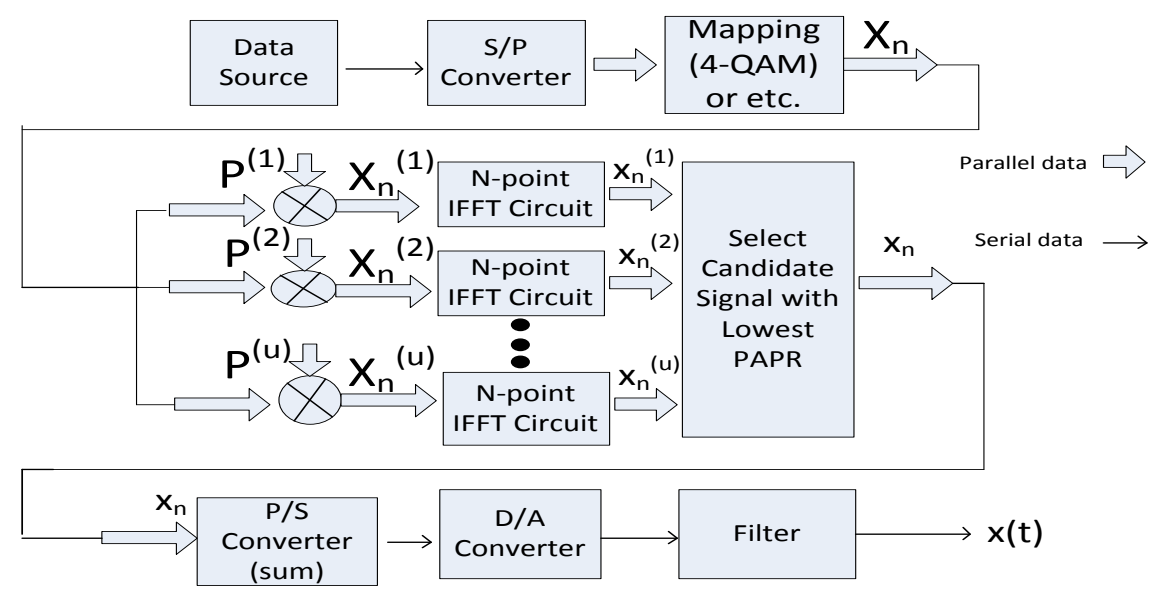

Fig.1 SPS-SLM based OFDM transmitter.

For example, let us consider, number of the sub carriers $=$ number of the IFFT points $=4$ and number of phase sequence $(\mathrm{U})=2$, then $\mathrm{D}=4,8,12,16,20,24 \ldots \ldots$, and $\mathrm{k}=0,1,2,3$. For the case when $\mathrm{u}=1$ output of the successive sections will be as follows:

$$
\mathrm{P}^{(1)}=\left[\mathrm{p}_{0}{ }^{(1)}, \mathrm{p}_{1}{ }^{(1)}, \mathrm{p}_{2}{ }^{(1)}, \mathrm{p}_{3}{ }^{(1)}\right]^{\mathrm{T}}, \mathrm{X}_{\mathrm{n}}=\left[\mathrm{X}_{0}, \mathrm{X}_{1}, \mathrm{X}_{2}, \mathrm{X}_{3}\right]^{\mathrm{T}}, X_{n}^{1}=\left[\mathrm{X}_{0} \mathrm{P}_{0}{ }^{(1)}, \mathrm{X}_{1} \mathrm{P}_{1}{ }^{(1)}, \mathrm{X}_{2} \mathrm{P}_{2}{ }^{(1)}, \mathrm{X}_{3} \mathrm{P}_{3}{ }^{(1)}\right]^{\mathrm{T}} \text { and } \mathrm{P}_{\mathrm{k}}{ }^{(1)}=\exp [\mathrm{j}
$$
$\{2 \pi \mathrm{k}(1-1) / \mathrm{D}+2 \pi(1-1) / \mathrm{D}\}]$.

And for $\mathrm{u}=2$ outputs of the same sections will be as follows:

$$
\mathrm{P}^{(2)}=\left[\mathrm{p}_{0}{ }^{(2)}, \mathrm{p}_{1}{ }^{(2)}, \mathrm{p}_{2}{ }^{(2)}, \mathrm{p}_{3}{ }^{(2)}\right]^{\mathrm{T}}, \mathrm{X}=\left[\mathrm{X}_{0}, \mathrm{X}_{1}, \mathrm{X}_{2}, \mathrm{X}_{3}\right]^{\mathrm{T}}, X_{n}^{2}=\left[\mathrm{X}_{0} \mathrm{P}_{0}{ }^{(2)}, \mathrm{X}_{1} \mathrm{P}_{1}{ }^{(2)}, \mathrm{X}_{2} \mathrm{P}_{2}{ }^{(2)}, \mathrm{X}_{3} \mathrm{P}_{3}{ }^{(2)}\right]^{\mathrm{T}} \text { and } \mathrm{P}_{\mathrm{k}}{ }^{(2)}=\exp [\mathrm{j}
$$
$\{2 \pi \mathrm{k}(2-1) / \mathrm{D}+2 \pi(2-1) / \mathrm{D}\}]$.

So a set of different OFDM data blocks $X_{n}^{(u)}=X_{n}^{2}=\left[\mathrm{X}_{0} \mathrm{P}_{0}{ }^{(\mathrm{u})}, \mathrm{X}_{1} \mathrm{P}_{1}{ }^{(\mathrm{u})}, \mathrm{X}_{2} \mathrm{P}_{2}{ }^{(2)}, \mathrm{X}_{3} \mathrm{P}_{3}{ }^{(\mathrm{u})} \ldots \ldots \ldots . . \mathrm{X}_{\mathrm{N}-1} \mathrm{P}_{\mathrm{N}-1}{ }^{(\mathrm{u})}\right]^{\mathrm{T}}$ are formed. Where $X_{n}^{(u)}=X_{n} \cdot P^{(u)}, n=0,1,2,3 \ldots N-1$ and $\mathrm{u}=0,1,2 \ldots \ldots \ldots . \mathrm{U}$. In the next step frequency-domain symbol vector $X_{n}^{(u)}$ is converted into time-domain symbol vector $x_{n}^{(u)}$ by IFFT operation. Mathematically it can be denoted as $x_{n}^{(u)}=$ IFFT $\left\{X_{n}^{(u)}\right\}^{\mathrm{T}}$ and finally select the one from $x_{n}^{(u)}$ which has a minimum PAPR and transmitted that after passing through parallel to serial $(\mathrm{P} / \mathrm{S})$ converter, digital to analog 
converter (DAC) and filter.

The model of SPS-SLM based OFDM receiver is shown in figure (2). The anti SPS-SLM based OFDM receiver receives the transmitted OFDM signal, passes it through the filter to remove noise. The filtered signal then passes through $\mathrm{A} / \mathrm{D}$ and $\mathrm{P} / \mathrm{S}$ converter. P/S converter provide the time-domain symbol vector $x_{n}^{(u)}$. This time-domain symbol vector again converted to frequency-domain symbol vector using FFT operation. Mathematically it can be written as $X_{n}^{(u)}=F F T\left(x_{n}^{(u)}\right)=\left[\mathrm{X}_{0} \mathrm{P}^{(\mathrm{u})}{ }_{0}, \mathrm{X}_{1} \mathrm{P}^{(\mathrm{u})}{ }_{1}, \mathrm{X}_{2} \mathrm{P}^{(\mathrm{u})}{ }_{2} \ldots \ldots . . \mathrm{X}_{\mathrm{N}-1} \mathrm{P}^{(\mathrm{u})}{ }_{\mathrm{N}-1}\right]$. Now the number of $U$ different anti SPS denoted by $\left(-\mathrm{p}_{\mathrm{k}}{ }^{(\mathrm{v})}\right)$ which multiply with frequency domain symbol vector $X_{n}^{(u)}$ element by element. Mathematically it can be written as ${ }^{(v)} X_{n}^{(u)}=-\mathrm{P}_{\mathrm{K}}^{(\mathrm{v})} \cdot X_{n}^{(\mathrm{u})}=\left[-\mathrm{P}^{(\mathrm{v})}{ }_{0} \mathrm{X}_{0} \mathrm{P}^{(\mathrm{u})}{ }_{0},-\mathrm{P}^{(\mathrm{v})}{ }_{1}\right.$ $\left.\mathrm{X}_{1} \mathrm{P}^{(\mathrm{u})}{ }_{1},-\mathrm{P}^{(\mathrm{v})}{ }_{2} \mathrm{X}_{2} \mathrm{P}^{(\mathrm{u})}{ }_{2} \ldots \ldots .-\mathrm{P}^{(\mathrm{v})}{ }_{\mathrm{N}-1} \mathrm{X}_{\mathrm{N}-1} \mathrm{P}^{(\mathrm{u})}{ }_{\mathrm{N}-1}\right]$.

The characteristic of anti SPS can be described by the following equations:

$$
\begin{aligned}
& -\mathrm{P}^{(\mathrm{v})}=\mathrm{p}_{\mathrm{k}}^{(\mathrm{v})}=\exp \left(\mathrm{j} \phi_{\mathrm{k}}{ }^{(\mathrm{v})}\right) \\
& -\mathrm{P}_{\mathrm{k}}{ }^{(\mathrm{v})}=\exp [-\mathrm{j}\{2 \pi \mathrm{k}(\mathrm{v}-1) / \mathrm{D}+2 \pi(\mathrm{v}-1) / \mathrm{D}\}]
\end{aligned}
$$

where $\mathrm{v}=1,2,3$ U.

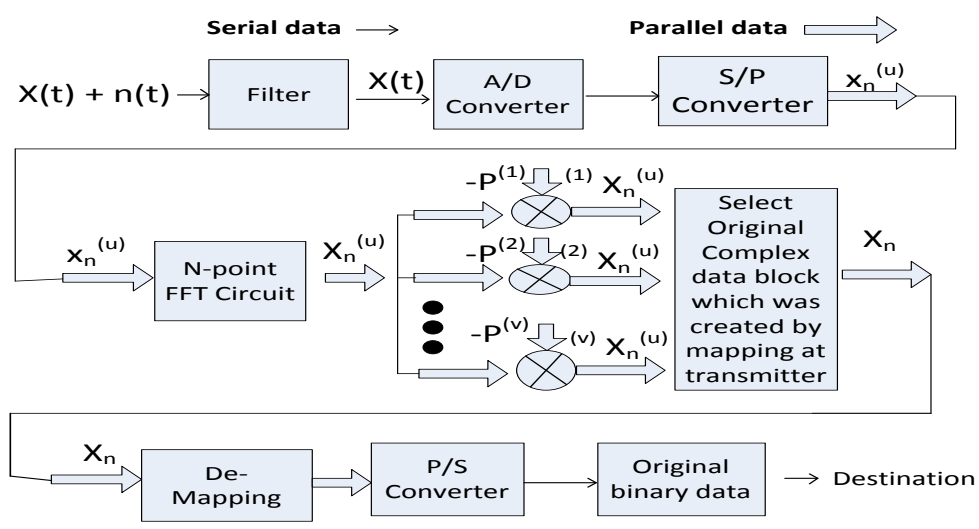

Fig.2 SPS-SLM based OFDM receiver

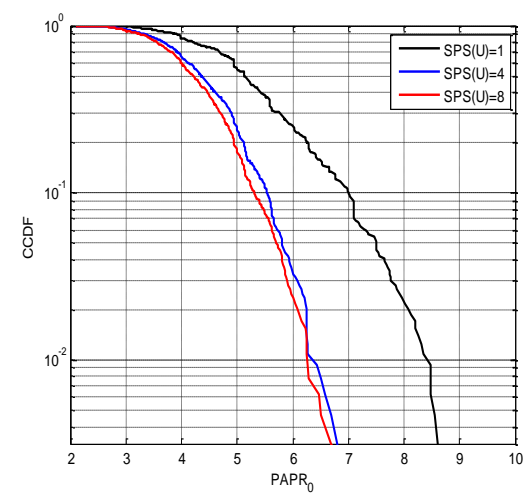

Fig.3 PAPR performance for number of subcarriers 16, modulation scheme 4QAM and phase divider (D) 32 . 
In notation ${ }^{(v)} X_{n}^{(u)}$, if the value of $\mathrm{v}$ is equal to the value of $\mathrm{u}$ then we get ${ }^{(\mathrm{v})} X_{n}^{(u)}=\mathrm{X}_{\mathrm{n}}$ which is selected by decision device in the receiver, where $v=1,2,3 \ldots \ldots \mathrm{U}$ and $\mathrm{u}=1,2,3,4 \ldots \ldots$. The complex frequency domain symbol vector $\mathrm{X}_{\mathrm{n}}$ converts into normal symbol vector (the vector was considered as data block in SPSSLM based OFDM transmitter). Now this vector or data block passes through P/S converter which gives the output data block of serial N symbol and so on. Finally every serial symbol in every data block converted into binary serial data which is the replica of the transmitted data.

\section{Results}

Performance of OFDM system depends on many factors; such as- number of subcarriers, number of phase sequences, modulation scheme used, type of channels, no. of phase divider etc. Performance study is generally done by changing one of the above parameters keeping others unchanged. In this paper the performance of the SPS-SLM technique having no side bands in reducing PAPR for different number of subcarriers have been studied. We have taken 16 and 32 subcarriers for 4QAM and 16QAM modulation schemes and for each case, observed the effect of changing phase sequences on PAPR reduction. In order to evaluate the performance of the system, a MATLAB simulation has been performed assuming nonlinear additive white Gaussian noise (AWGN) channel and using randomly generated data bits.

Fig. (3) presents the plot of complementary cumulative distribution function (CCDF) of PAPR of OFDM signal for 16 subcarriers. From the figure it is observed that for pure OFDM $(u=1)$ i.e., when no PAPR reduction measure is applied, PAPR of OFDM signals is about $8.6 \mathrm{~dB}$. PAPR of OFDM signal is $6.8 \mathrm{~dB}$ and $6.7 \mathrm{~dB}$ for number of phase sequences 4 and 8 respectively.

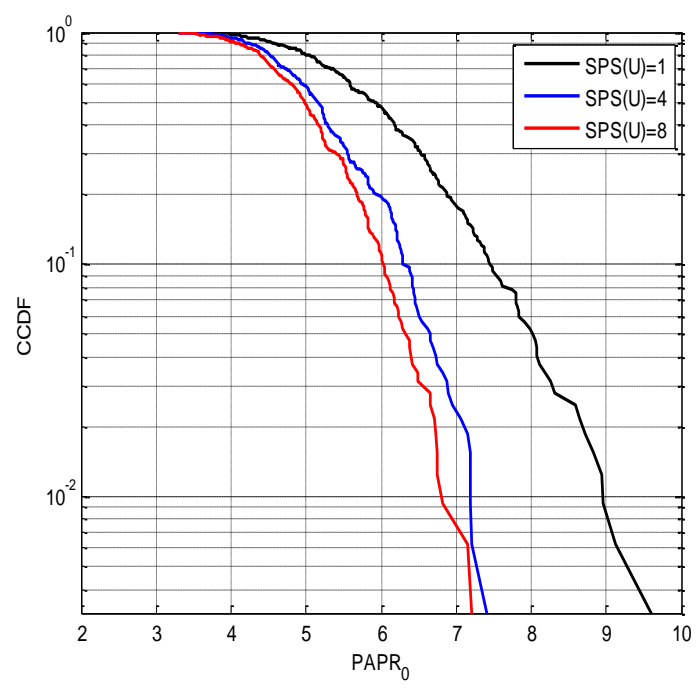

Fig.4 PAPR performance for number of subcarriers 32, modulation scheme 4QAM and phase divider (D) 64 . 


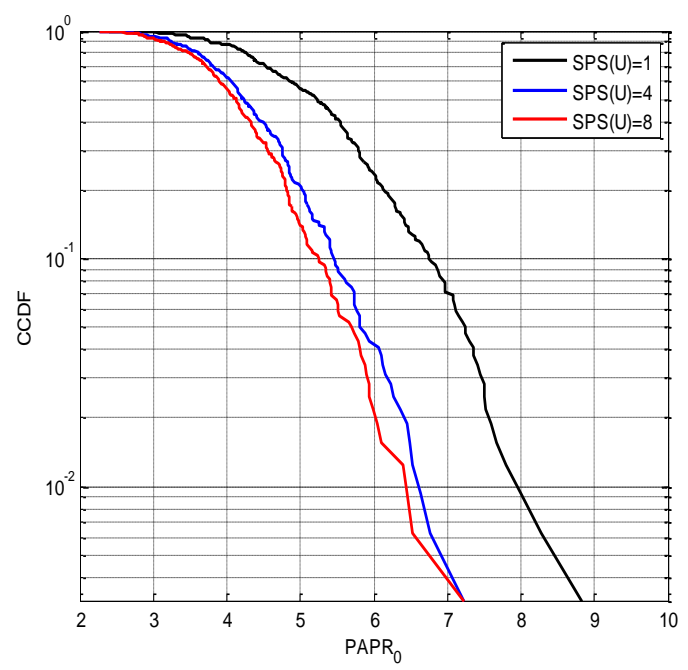

Fig.5 PAPR performance for number of subcarriers 16, modulation scheme 16QAM and phase divider (D) $=32$

Fig. (4) depicts the plot of CCDF of PAPR of OFDM signal for 32 subcarriers. In this case for original OFDM, PAPR of OFDM signal is about $9.6 \mathrm{~dB}$. For $\mathrm{u}=4$ and $\mathrm{u}=8$ PAPR of OFDM signal is $7.4 \mathrm{~dB}$ and $7.2 \mathrm{~dB}$ respectively. Fig.(5) gives the plot of CCDF of PAPR of OFDM signal for 16QAM modulation scheme. In this case number of subcarriers is 16. For this scheme, for original OFDM, PAPR of the signal is about $8.8 \mathrm{~dB}$. PAPR value about $7.2 \mathrm{~dB}$ has been observed for phase sequences 4 and 8 .

\section{Conclusions}

This paper discusses the implementation of SPS-SLM OFDM system omitting the side information and its performance evaluation regarding PAPR. In this study PAPR was seen to reduce up to 7.0dB from about 9.0 $\mathrm{dB}$ for pure OFDM when no PAPR reduction technique is utilized. Also it was seen that PAPR reduces rapidly as the number of phase sequences was increased from 1 to 4 but further increment of phase sequences have small effect on PAPR reduction. It was observed from this study that there is little change in PAPR when modulation technique was changed from 4QAM to 16QAM.

Paper [25] describes an ODFM technique without side information. In that paper a cyclic Hadamard matrix was utilized to modify the transmitted data in $U$ independent form that eliminated the side information. To study the performance of the method in reducing PAPR and bit error rate (BER) a random-like irregular repeat accumulate encoder was utilized. In this study it was observed that for pure OFDM $(u=1)$ PAPR level was 9.5 $\mathrm{dB}$ which reduces to $7.0 \mathrm{~dB}$ for number of phase sequences 4 .

A simple SLM technique requires transmission of no extra bits has been described in paper [26]. Instead of transmitting side information as additional bits this method embedded the side information bits into the transmitted frame at a specific position. The locations of the extended symbols depend on the values of the side information bits. In the receiver side, the side information bits are detected by finding the correct locations. In this technique $3.6 \mathrm{~dB}$ of PAPR reduction at the CCDF level of $10^{-4}$ was achieved which was in the same level of classical SLM. 


\section{References}

[1] $\mathrm{Wu}, \mathrm{Y}$. and W. Y. Zou, "Orthogonal frequency division multiplexing: A multi-carrier modulation scheme," IEEE Trans. Consumer Electronics, vol. 41, no. 3, pp. 392-399, 1995.

[2] Zou, W. Y. and Y. Wu "COFDM: An overview" IEEE Trans. Broadcasting, vol. 41, no. 1, pp. 1-8, 1995.

[3] Bauml, R. W.; R. F. H. Fischer and J. B. Huber, "Reducing the peak -to- average power ratio of multicarrier modulation by selected mapping", Electron. Lett., vol. 32, no. 22, pp. 2056-2057, 1996.

[4] Han, S. H.; J. M. Ciof and J. H. Lee, "Tone injection with hexagonal constellation for peak-to-average power ratio reduction in OFDM" IEEE Commun. Lett., vol. 10, no. 9, pp. 646-648, 2006.

[5] Jones, A.E.; T. A. Wilkinson, and S. K. Barton, "Block coding scheme for reduction of peak to mean envelope power ratio of multicarrier transmission scheme", Electron. Lett., vol. 30, no. 25, pp. 2098-2099, 1994

[6] Krongold, B. S. and D. L. Jones "PAR reduction in OFDM via active constellation extension", IEEE Trans. Broadcast., vol. 49, no. 3, pp. 258- 268, 2003.

[7] Li, X. and L. J. Cimini, Jr., "Effect of clipping and filtering on the performance of OFDM", IEEE Commun. Lett., vol. 2, no. 5, pp. 131-133, 1998.

[8] Ochiai, H. and H. Imai, "Performance of the deliberate clipping with adaptive symbol selection for strictly band-limited OFDM systems", IEEE J. Sel. Areas Commun., vol. 18, no. 11, pp. 2270-2277, 2000.

[9] Ochiai, H. and H. Imai, "Performance analysis of deliberately clipped OFDM signals", IEEE Trans. Commun., vol. 50, no. 1, pp. 89-101, 2002.

[10] Breiling, M. and S. H. Müller-Weinfurtner and J. B. Huber, "SLM peak-power reduction without explicit side information", IEEE Commun. Lett., vol. 5, no. 6, pp. 239-241, 2001.

[11] Kim, H.; E. Hong; C. Ahn and D. Har "A Pilot Symbol Pattern Enabling Data Recovery Without Side Information in PTS-Based OFDM Systems", IEEE Transactions on Broadcasting, vol- 07, 2011.

[12] Jayalath, A. D. S. and C. Tellambura, "SLM and PTS peak-power reduction of OFDM signals without side information", IEEE Trans. Wireless Commun., vol. 4, no. 5, pp. 2006-2013, 2005.

[13] Kasiri, K. and M. J. Dehghani1, "A Blind SLM Scheme for Reduction of PAPR in OFDM Systems", World Academy of Science, Engineering and Technology, vol-50, 2009.

[14] Han, S.H. and J.H. Lee, "An Overview of Peak-to-Average Power Ratio Reduction Techniques For Multicarrier Transmission”, IEEE Wireless Communications, vol.12, no.2, pp.56-65, 2005.

[15] Jiang, T. and Y. Wu, "An Overview: Peak-to-Average Power Ratio Reduction Techniques for OFDM Signals" IEEE Transactions on Broadcasting, Vol.54, No.2, pp.257-268, June 2008.

[16] Armstrong, J. "Peak-to-Average Reduction for OFDM by Repeated Clipping and Frequency Domain Filtering" IEEE Electronics Letters, vol.38, No.5, pp.246-247, 2002.

[17] Rao, K.D. and T.S.N. Murthy, "Analysis of Effects of Clipping and Filtering on the Performance of MBOFDM UWB Signals", Proc. of the 2007 15th International Conference on Digital Signal Processing (DSP 2007), IEEE, pp.559-562.

[18] Ghassemi, A. and T.A. Gulliver "PAPR Application of OFDM Using PTS and Error-Correcting Code Sub- blocking" IEEE Trans. On Wireless Communications, Vol.9, No.3, pp. 980-989, March 2010.

[19] Goff, S.Y. Le; S. Al-Samahi; .S.B. Khoo, C.C.Tsimenidis and B.S.Sharif "Selected Mapping without side information for PAPR Reduction in OFDM" IEEE Transactions on Wireless Communications, Vol.8, No.7, July 2009, pp.3320-3325.

[20] Han, S.H. and J.H. Lee, "PAPR Reduction of OFDM Signals using a Reduced Complexity PTS Technique" IEEE Signal Processing Letters, vol.11, no.11, pp.887-890, 2004.

[21] Hill, G. and M. Faulkner, "Comparison of Low Complexity Clipping Algorithms for OFDM" IEEE International Symposium on Personal, Indoor and Mobile Radio communications, 2002,Vol.1, pp.227- 
231.

[22] Yusof, S.K. and N. Fisal, "Correlative Coding with Clipping and Filtering Technique in OFDM Systems" ICICS-PCM 2003, Singapore, IEEE 2003, pp.1456-1459.

[23] Taha, Z.Q. and X. Liu "An Adaptive Coding Technique for PAPR Reduction" Global Telecommunication conference, Globecom 2007, IEEE, pp.376-380.

[24] Paterson, K. "Generalized Reed-Muller codes and power control in OFDM modulation" IEEE Trans. Inform. Theory, vol. 46, no. 1, pp. 104-120, Jan. 2000.

[25] Kargozar, F.; M. Nilashi; O. Ibrahim and M. Barisami "A novel PAPR reduction scheme based on selective mapping and a random-like coding with no explicit side information in OFDM" J. of Information Engineering and Applications, Vol-1, No. 1, 2011.

[26] Goff, Y. Le.; C.C. Tsimenidis and B.S. Sharif "OFDM PAPR reduction using selected mapping without side information", IEEE International conference on communications 2007 (ICC 2007), 24-28 June SECC in Glasgow, Scotland. DoI: 10.1109/ICC.2007.716.

\section{Author(s) Profile}

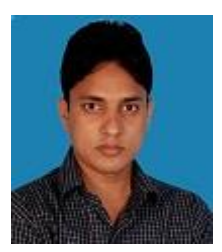

system.
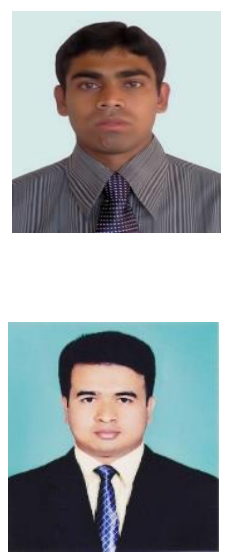

Md. Khalid Hossain Jewel received his Bachelor's and Master's degree from the dept. of Applied Physics, Electronics \& Communication Engineering of Islamic University, Kushtia7003, Bangladesh in 2004 and 2005 respectively. Now he is currently an assistant professor in the same department .His research interest includes Ad-hoc wireless communication, cellular mobile communication and optical fiber communication.

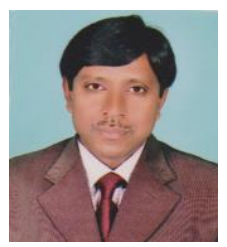

Md. Shahjahan Ali received B.Sc (Hons) and M.Sc in 1988 and 1989 respectively in Applied Physics and Electronics from Rajshahi University, Bangladesh. He received Ph.D. in engineering and technology from Dhaka University in 2011. Currently Dr. Ali is a professor in the department of Applied Physics, Electronics \& Communication Engineering, Islamic University, Kushtia-7003, Bangladesh. His research interests include modeling and optimization of wireless network and applications of remote sensing technology. 